\title{
COMPOSIÇÃO QUÍMICA, ATIVIDADE CITOTÓXICA E ANTIOXIDANTE DE UM TIPO DE PRÓPOLIS DA BAHIA $^{\#}$
}

\author{
Darlan C. dos Santos ${ }^{\mathrm{a}, \mathrm{b}}$, Jorge Mauricio David ${ }^{\mathrm{b}, *}$ e Juceni Pereira David ${ }^{\mathrm{c}}$ \\ ${ }^{a}$ Colegiado de Ciências Naturais, Universidade do Estado do Amapá, Av. Presidente Vargas, 650 68900-070 Macapá - AP, Brasil \\ 'Instituto de Química, Universidade Federal da Bahia, Campus Ondina 40170-280 Salvador - BA, Brasil \\ ${ }^{c}$ Faculdade de Farmácia, Universidade Federal da Bahia, Campus Ondina, 40170-280 Salvador - BA, Brasil
}

Recebido em 22/06/2016, aceito em 12/09/2016, web em 29/09/2016

\begin{abstract}
CHEMICAL COMPOSITION, CYTOTOXIC ACTIVITY AND ANTIOXIDANT OF A PROPOLIS TYPE FROM BAHIA. Propolis is a complex mixture composed by salivary secretions of bees and a resinous and balsamic material collected by them from different part of plants. This material is well employed by people due its antimicrobial, antioxidant, anti-inflammatory, antiviral properties beside others. However, the chemical composition varies according the habitat and season. This work describes the identification of saturated hydrocarbons, methyl cinnamate, sitosterol cinnamate and ananixanthone isolated from the brown propolis produced in Bahia, Brazil, classified as Propolis Type 6. Besides, antioxidant activities and cytotoxicity of the extracts were evaluated by the DPPH quenching and Brine Shrimp Test. The extracts showed weak antioxidant activities and high cytotoxicity, especially the methanol and chloroformic extracts.
\end{abstract}

Keywords: Propolis; ananixanthone; sitosterol cinnamate

\section{INTRODUÇÃO}

A própolis é uma mistura complexa produzida pela abelhas a partir de diversas substâncias coletadas de diversas partes das plantas como botões florais, exsudatos e brotos. ${ }^{1}$ As abelhas utilizam a própolis para proteger a colmeia contra agente invasores, embalsamar animais, manter a temperatura agradável e para o preparo de locais assépticos para a postura da abelha rainha. ${ }^{2}$ Sua coloração é variável e depende da região que foi coletada, seu odor é característico e variável, entretanto algumas amostras não possuem odor, apresenta ponto de fusão na faixa de $60-70{ }^{\circ} \mathrm{C}$, sendo que alguns casos seu ponto de fusão pode chegar até $100^{\circ} \mathrm{C} .^{2}$

Há relatos da utilização da própolis na medicina popular desde a antiguidade. Na Idade Média era comum utilizar o própolis no umbigo dos bebês para fins de cicatrização. ${ }^{3}$ Durante a Segunda Guerra Mundial era utilizada em clínicas russas no tratamento de ferimentos e, foi amplamente utilizada na antiga URSS tanto na medicina humana quanto na medicina veterinária no tratamento da tuberculose. ${ }^{4}$ Diversas atividades biológicas já foram relatadas à própolis tais como antioxidante, antitumoral, anti-inflamatória, antibacteriana, antiviral e antifúngica. ${ }^{5}$ Comercialmente é encontrada em várias preparações como comprimidos, pastilhas, dentifrícios, loções, cremes faciais, tinturas, pomadas. ${ }^{6}$

A composição química da própolis é variável e depende da variedade genética da abelha que a produziu ${ }^{7}$ e da biodiversidade da região visitada pelas abelhas. ${ }^{8}$ Os compostos fenólicos, com a predominância dos flavonoides, são os compostos que são isolados com mais frequência em amostras de própolis, ${ }^{9}$ entretanto já foram relatados triterpenoides, benzofenonas, esteroides, ácidos graxos, hidrocarbonetos, cetonas, aminoácidos, vitaminas $\mathrm{A}, \mathrm{B}_{1}, \mathrm{~B}_{2}, \mathrm{~B}_{6}, \mathrm{C}$ e E e minerais como $\mathrm{Mg}, \mathrm{Cu}$, $\mathrm{Ca}, \mathrm{Al}, \mathrm{V}, \mathrm{Ni}, \mathrm{Zn}, \mathrm{Cr} .{ }^{10}$ Este trabalho descreve a avaliação da atividade antioxidantes e citotóxica da própolis produzida na Bahia bem como a composição química do extrato hexânico.

*e-mail: darlanquimico@ hotmail.com; jmdavid@ufba.br

"This paper is part of the PubliSBQ Special Issue in honor of the late Prof. Angelo da Cunha Pinto.

\section{PARTE EXPERIMENTAL}

\section{Procedimentos experimentais gerais}

Os solventes utilizados no preparo dos extratos e nas eluições cromatográficas (hexano, acetato de etila, diclorometano, clorofórmio, metanol) foram de grau analítico marca Qhemis. Nos procedimentos de purificação por cromatografia em coluna (CC) os adsorventes utilizados como fase estacionária foram gel de sílica 60 da Merck e Acros com diâmetro de partículas entre 0,063-0,200 mm e 0,040$0,063 \mathrm{~mm}$. Para a cromatografia em camada delgada comparativa (CCDC), foram utilizadas placas de $20 \times 20 \mathrm{~cm}$ de gel de sílica 60 $\mathrm{F}_{254}$ da Merck para reunir as frações oriundas da cromatografia em coluna. Para o isolamento de substâncias por cromatografia em camada delgada preparativa (CCDP) foram utilizadas placas de gel de sílica $60 \mathrm{~F}_{254}$ de $1 \mathrm{~mm}$ de espessura da Merck. As placas foram reveladas por radiação com luz UV (254 e $336 \mathrm{~nm}$ ) e vapores de iodo. Os espectros de $\mathrm{RMN}$ de ${ }^{1} \mathrm{H} \mathrm{e}{ }^{13} \mathrm{C}$ foram registrados em espectrometro da Varian, modelo GEMINI 2000 operando a $300 \mathrm{MHz}$ para hidrogênio e 75,5 $\mathrm{MHz}$ para ${ }^{13} \mathrm{C}$, utilizando-se $\mathrm{CDCl}_{3}$ como solvente e o sinal do resíduo de cloroformio em relação ao tetrametilsilano (TMS) como referência. Os cromatogramas foram obtidos em um cromatógrafo à gás acoplado à espectometria de massas de baixa resolução da Shimadzu, com detector seletivo de massas, modelo QP 2010. Para a realização das análises utilizou-se o método onde a temperatura do injetor foi $290^{\circ} \mathrm{C}$ com temperatura inicial de $50{ }^{\circ} \mathrm{C}$ por $1 \mathrm{~min}$, aumentando de $50^{\circ} \mathrm{C}$ a $180^{\circ} \mathrm{C}$ na razão de $15^{\circ} \mathrm{C} / \mathrm{min}$, e de $180{ }^{\circ} \mathrm{C}$ a $230{ }^{\circ} \mathrm{C}$ na razão de $7{ }^{\circ} \mathrm{C} / \mathrm{min}$, e de $230{ }^{\circ} \mathrm{C}$ a $250{ }^{\circ} \mathrm{C}$ na razão de $15{ }^{\circ} \mathrm{C} / \mathrm{min}$, permanecendo nessa temperatura por $10 \mathrm{~min}$. Em seguida, a temperatura passou de $250{ }^{\circ} \mathrm{C}$ para $285^{\circ} \mathrm{C}$ na razão de $4{ }^{\circ} \mathrm{C} / \mathrm{min}$. A temperatura final permaneceu em $285^{\circ} \mathrm{C}$ por $40 \mathrm{~min}$. A temperatura do detector foi de $290{ }^{\circ} \mathrm{C}$ e a temperatura na interface do sistema CG-EM foi de $290^{\circ} \mathrm{C}$. O detector de massas operou com ionização por impacto de elétrons $(70 \mathrm{eV})$ e varredura de massas entre o intervalo de 35 a $1000 \mathrm{Da}$. 


\section{Coleta da própolis}

A própolis marrom foi cedida pelo Prof. Ednildo Andrade Torres da Universidade Federal da Bahia diretamente na colmeia das abelhas Apis melifera, em janeiro de 2008, na apícola localizada na fazenda experimental Naturapi no município de Entre Rios, Bahia.

\section{Extração e isolamento dos constituintes}

A amostra de própolis $(141,18 \mathrm{~g})$ foi submetida à extração através de maceração por 48 horas utilizando $2 \mathrm{~L}$ de hexano como líquido extrator. $\mathrm{O}$ procedimento foi realizado por três vezes consecutivas, e o filtrado obtido em cada etapa foi reunido e concentrado sob pressão reduzida, obtendo-se assim o extrato hexânico da própolis $(34,91 \mathrm{~g})$. As tortas obtida após extração com hexano foram posteriormente submetidas à maceração com $2 \mathrm{~L}$ de $\mathrm{MeOH}$, três vezes consecutivas, com cerca de $48 \mathrm{~h}$ cada, e o filtrado obtido em cada etapa foi reunido e concentrado sob pressão reduzida, originando o extrato metanólico da própolis que foi dissolvido em $\mathrm{MeOH} / \mathrm{H}_{2} \mathrm{O}(7 / 3)$ e particionado com clorofórmio, dando origem a fases clorofórmica $(53,22 \mathrm{~g})$ e hidrometanólica (2,2 g).

$\mathrm{O}$ extrato hexânico foi submetido a fracionamento por CC sob sílica gel 60 utilizando-se inicialmente hexano como fase móvel e em seguida misturas de hex/AcOEt em gradiente de polaridade. Foram obtidas 38 frações que foram agrupadas após análise em CCDC. A fração 1, foi submetida à análise por CG-EM para identificação dos componetes da mistura. A fração reunida $6(64 \mathrm{mg})$ coletada em hex/ AcOEt (8:2) submetida submetido à fracionamento por $\mathrm{CC}$ de sílica gel 60, utilizando a misturas de hex/AcOEt em gradiente de polaridade como fase móvel foi possível isolar o composto 5 (15 mg). A fração reunida 7, coletada em hex/AcOEt (7:3), forneceu composto $6(13 \mathrm{mg})$ após separação por cromatografia em camada delgada preparativa CCDP, utilizando-se hex/AcOEt (8:2) como fase móvel.

A fase clorofórmica, 53,22 g, até o presente momento, submetida à cromatografia em coluna de sílica gel 60 como fase estacionária e fase móvel mistura de hex/AcOEt em gradiente crescente de polaridade forneceu 37 frações de $200 \mathrm{~mL}$ cada, que foram reunidas em 20 frações após serem analisadas por CCDC. A partir da fração reunida 5 foi possível isolar 7 (18 mg) após separação por CCDP utilizando-se a mistura de hex/AcOEt (9:1) como fase móvel.

\section{Ensaio de sequestro de radicais DPPH}

Os extratos metanólico e hexânico da própolis foram submetidos a teste de atividade antioxidante in vitro, utilizando-se a metodologia do sequestro de radical DPPH (2,2-difenil-1-picrilhidrazila). ${ }^{11}$

Preparou-se $50 \mathrm{~mL}$ de uma solução metanólica de DPPH ( $45 \mu \mathrm{g} \mathrm{mL} \mathrm{m}^{-1}$ ) em seguida preparou-se soluções metanólicas dos extratos em 5 concentrações $\left(20,50,100,150,200 \mu \mathrm{g} \mathrm{m}^{-1}\right)$, sendo que o ácido gálico foi utilizado como padrão. Para realização dos testes foram utilizados $3 \mathrm{~mL}$ de solução de DPPH com $1 \mathrm{~mL}$ da amostra, após 15 minutos de incubação as absorvâncias foram medidas a $517 \mathrm{~nm}$ em um espectrofotômetro de microplacas (Biotek, USA). Para zerar o equipamento preparou-se o branco com $3 \mathrm{~mL}$ de DPPH e $1 \mathrm{~mL}$ de metanol. Todas as reações foram realizadas a temperatura ambiente e em local protegido da luz. Todas as análises foram realizadas em triplicatas. $\mathrm{O} \mathrm{IC}_{50}$ foi calculado utilizando software ED50v10 para Microsoft Excel Add Ins fornecido pelo espectrofotometro.

\section{Bioensaio de letalidade dos extratos sobre Artemia salina}

O ensaio de toxicidade sobre Artemia salina Leach foi realizado de acordo com a metodologia descrista na literatura. ${ }^{12}$ Foram preparadas 5 soluções de $\left(50,75,100,150\right.$ e $\left.200 \mu \mathrm{g} \mathrm{mL}^{-1}\right)$ dos extratos hexânico, metanólico e clorofórmico da própolis.

Os ovos de Artemia salina foram eclodidos em um aquário retangular, utilizando-se solução salina $3,8 \mathrm{~g} \mathrm{~L}^{-1}$ obtida de acordo com as especificações do fabricante. $\mathrm{O}$ sistema foi aerado com bomba específica. $\mathrm{O}$ aquário foi construído com uma divisão interna de acrílico contendo vários orifícios, produzindo dois compartimentos não equivalentes em tamanho. Os ovos foram adicionados no compartimento menor, previamente escurecido utilizando-se papel alumínio. O compartimento maior foi externamente iluminado com lâmpada $60 \mathrm{~W}$, de modo a atrair os náuplios de Artemia para este compartimento, após a eclosão.

Após 48 horas foram transferidos 10 náuplios para cada frasco, utilizando pipeta Pasteur esterilizada e, o volume dos frascos completados até $5 \mathrm{~mL}$ com água do mar artificial. As amostras bem como os controles foram mantidos sob iluminação por 24 horas e o número de organismos sobreviventes foram determinados com auxílio de uma lupa. Todos os experimentos, inclusive os de controle foram realizados em triplicata. Os dados obtidos foram processados e os valores de $\mathrm{CL}_{50}$ foram calculados usando o método de Probit.

\section{Cinamato de sitosterila, 5}

$\mathrm{RMN}{ }^{13} \mathrm{C}\left[75 \mathrm{MHz}, \mathrm{CDCl}_{3} \delta\right.$ (ppm)]: 166,41 (C-9'), 144,41 (C7') 139,69 (C-5), 134,54 (C-1'), 130,15 (C-4'), 128,85 (C-3' e C-5'), 128,03 (C-2' e C-6'), 122,72 (C-6), 118,71 (C-8'), 74,10 (C-3), 56,70 (C-14), 56,03 (C-17), 50,05 (C-9), 45,84 (C-24), 42,32 (C-4 e C-13), 39,73 (C-12), 37,03 (C-1), 36,63 (C-10), 36, 15 (C-20), 33, 94 (C-22), 33,70 (C-23), 31,92 (C-7 e C-8), 31,88 (C-2), 28,25 (C-16), 26,01 (C-25), 24,29 (C-15), 23,06 (C-28), 21,04 (C-11), 19,81 (C-27), 19,35 (C-19), 19,03 (C-21 e C-26), 11,86 (C-18 e C-29)

\section{Cinamato de metila, 6}

RMN $\left.{ }^{1} \mathrm{H}(300 \mathrm{MHz}) \mathrm{CDCl}_{3} \delta(\mathrm{ppm})\right]: 7,77(1 \mathrm{H}, \mathrm{d}, J=15,7 \mathrm{~Hz}$, H-3), 7,62 (1H, m, H-7), 7,50 (2H, m, H-6 e H-8), 7,36 (2H, m, H-5 e H-9), 6,52 (1H, d, $J=15,7 \mathrm{~Hz}, \mathrm{H}-2), 3,80\left(3 \mathrm{H}, \mathrm{s}, \mathrm{O}-\mathrm{CH}_{3}\right)$. RMN $\left.{ }^{13} \mathrm{C}(75 \mathrm{MHz}) \mathrm{CDCl}_{3} \delta(\mathrm{ppm})\right]: 167,39(\mathrm{C}-1), 144,84(\mathrm{C}-3), 134,32$ (C-4), 130,25 (C-7), 128,84 (C-6 e C-8), 128,02 (C-5 e C-9), 117,75 (C-2), 51,68 (C-10).

\section{Ananixantona, 7}

$\left.\mathrm{RMN}{ }^{1} \mathrm{H}(300 \mathrm{MHz}) \mathrm{CDCl}_{3} \delta(\mathrm{ppm})\right]: 13,20(1 \mathrm{H}, \mathrm{s}, \mathrm{C}-1, \mathrm{OH})$, $7,80(1 \mathrm{H}, \mathrm{dd}, J=7,5$ e $1,8 \mathrm{~Hz}, \mathrm{H}-8), 7,32$ ( $1 \mathrm{H}, \mathrm{dd}, J=6,3$ e 1,8, H-6), $7,26(1 \mathrm{H}, \mathrm{t}, J=7,5 \mathrm{~Hz}, \mathrm{H}-7), 6,82(1 \mathrm{H}, \mathrm{d}, J=10, \mathrm{H}-11), 5,66(1 \mathrm{H}, \mathrm{d}$, $J=10, \mathrm{H}-12), 5,26(1 \mathrm{H}, \mathrm{t}, J=2,0, \mathrm{H}-17), 3,38(2 \mathrm{H}, \mathrm{d}, J=6,9, \mathrm{H}-16)$, 1,80( $3 \mathrm{H}, \mathrm{s}, \mathrm{H}-19), 1,69$ (3H,s, H-20), 1,50 (3H, s, H-14 ou H-15), 1,25 (3H, s, H-14 ou H-15). RMN $\left.{ }^{13} \mathrm{C}(75 \mathrm{MHz}) \mathrm{CDCl}_{3} \delta(\mathrm{ppm})\right]$ : 180,96 (C-9), 160,74 (C-1), 159.00 (C-3), 149,85 (C-4a), 144,34 (C-5 ou C-10a), 132,10 (C-18), 127,30 (C-12), 123,89 (C-7), 122,15 (C-2), 121,88 (C-17), 121,15 (C-8a), 120,17 (C-6), 117,02 (C-8a), 115,03 (C-11) 103,60 (C-9a), 100,67 (C-4), 78,05 (C-13), 28,15 (C-14 e C-15), 25,80 (C-19), 21,21 (C-16), 17,90 (C-20).

\section{RESULTADOS E DISCUSSÃO}

No cromatograma de íons totais obtido por CG (Figura 1) da fração 5 foram registrados quatro picos majoritários com diferentes tempos de retenção, e cada um deles originou um espectro de massas. O pico em 18,5 min. originou um íon molecular $\mathrm{m} / \mathrm{z}$, 212, o pico em 20,7 min. originou um íon molecular $\mathrm{m} / \mathrm{z} 226$, o pico em $24 \mathrm{~min}$. originou um íon molecular $\mathrm{m} / \mathrm{z}, 240$ e o pico em $34,5 \mathrm{~min}$. originou um íon molecular $m / z 324$, esses íons moleculares correspondem ao pentadecano $\mathbf{1}$, hexadecano 2 , heptadecano 3 e ao tricosano 4 .

A identificação dos hidrocarbonetos foi realizada através da 


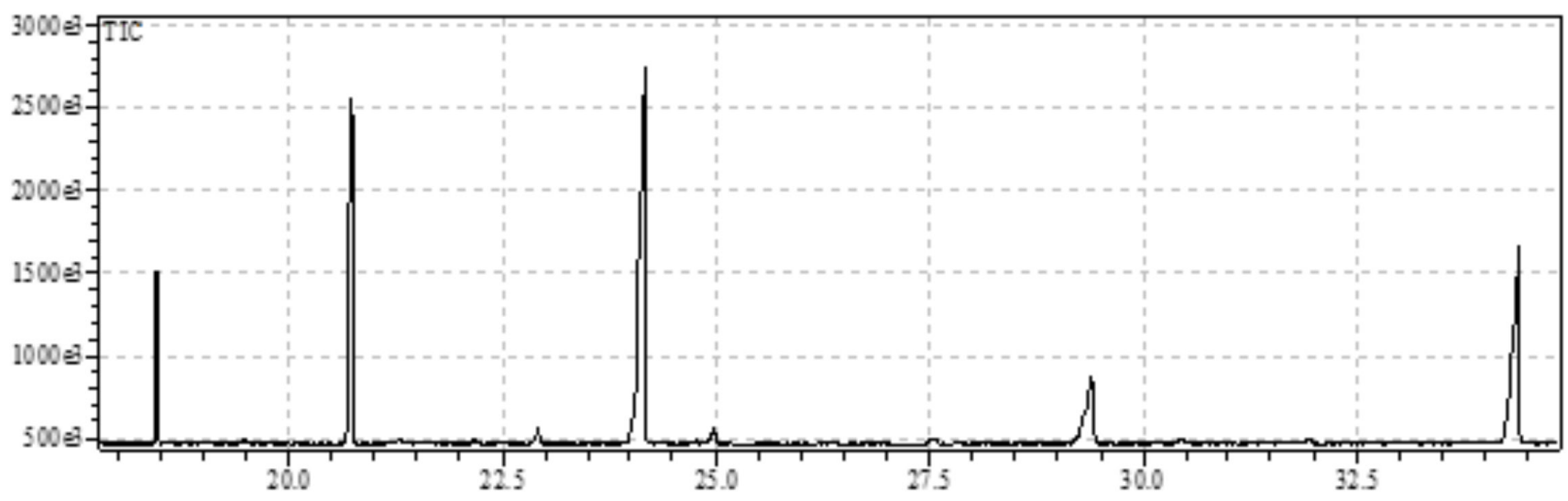

Figura 1. Cromatograma de íons totais da fração obtida do extrato hexânico da própolis da Bahia

avaliação dos íons moleculares, indice de retenção e fragmentações e com a comparação dos espectros de massas obtidos com os da biblioteca NIST 147 e WILEY 8 com similaridade acima de 95\%. Esses compostos são comumente encontrados em ceras de abelhas, além disso, já foram identificados em diversos tipos de própolis. Desta forma, pode-se admitir que a provável composição de compostos alifáticos presentes no extrato hexânico seja a mistura desses hidrocarbonetos.

$\mathrm{O}$ espectro de $\mathrm{RMN}{ }^{1} \mathrm{H}$ do composto 5 apresentou sinais na região entre $\delta 0,70$ a 1,26 característicos aos grupos metílicos do sistema esteroidal $\left(\mathrm{CH}_{3}-18, \mathrm{CH}_{3}-19, \mathrm{CH}_{3}-21, \mathrm{CH}_{3}-26, \mathrm{CH}_{3}-27\right.$, $\mathrm{CH}_{3}$-29). A presença de um multipleto em $\delta 4.75$ indica a presença de um hidrogênio oximetínico que, pelo deslocamento indica que a hidroxília encontra-se esterificada, pois o valor de H-3 no sitosterol é em campo mais baixo $(\delta \sim 3,3)$. O dubleto em $\delta 5,41(J=4,5 \mathrm{~Hz})$ é característico de hidrogênio olefínico $\mathrm{H}-6$ do sistosterol. Os dubletos em $\delta$ 6,40 e 7,65 apresentando $J=15,9 \mathrm{~Hz}$ são indicativos de sistema olefínico com configuração trans e os sinais na região entre $\delta 7,37$ e 7,70 , integrando para $5 \mathrm{H}$ indicam a presença de um anel aromático monossubstituido.

O espectro de RMN de ${ }^{13} \mathrm{C}$ corrobora a existência de um carbono oximetínico em $\delta 74,10$, que também encontra-se mais desblindado quando comparado com o C-3 do $\beta$-sitosterol devido esterificação com o grupo cinamoila. Os carbonos olefínicos em $\delta 139,41$ e 122,71 referentes à (C-5 e C-6), respectivamente, corroboram com a estrutura esteroidal da substância. O registro de um sinal em $\delta 166,41$ referente à carbono acila, confirma que a substância é um derivado esterificado do ácido cinâmico com sitosterol. Além disso, estes dados comparados a valores descritos na literatura ${ }^{13}$ do $\beta$-sitosterol permitiram a identificação inequívoca da substância como sendo o cinamato de sitosterila (Figura 2).

A literatura registra que o cinamato de sitosterila possui atividade alelopática. Este composto foi isolado anteriormente de Lafoensia glyptocarpa (Lytraceae), ${ }^{14}$ porém esse é o primeiro relato dessa substância na própolis. Apesar de ser uma substância já conhecida seus dados de RMN, ainda não estavam descritos na literatura.

$\mathrm{O}$ espectro de $\mathrm{RMN}$ de ${ }^{1} \mathrm{H}$ do trans cinamato de metila 6 , apresentou um sinal para um grupo metoxila em $\delta 3,80$ e um par de dubletos em 6,50 $(J=15,7)$ e 7,80 $(J=15,7)$ equivalentes a uma dupla ligação de configuaração trans. Os sinais em $\delta 7,36,7,50$ e 7,62, multiplicidade e constantes de acoplamento indicam a presença de uma anel aromático monossubstituído. $\mathrm{O}$ espectro de ${ }^{13} \mathrm{C}$ exibe um sinal em $\delta 51,68$ característico de grupo metoxílico ligado a carbono acila. A presença de um carbono acila foi corfimada através do sinal em $\delta 167,39$ típico de ésteres conjugados. A natureza aromática foi confirmada através dos sinais $\delta 128,02$ (C-5 e C-9), 128,84 (C-6 e
C-8), 130,25 (C-7) e 134,32 (C-4), desta forma foi possível identificar a substância como sendo o trans cinamato de metila (Figura 2) cujo os dados espectroscópicos estão de acordo com os dados descristos na literatura. ${ }^{15} \mathrm{O}$ trans cinamato de metila já havia sido identificado utilizando-se a técnica CG-MS em própolis tipo 1 oriunda da região do sul do Brasil, ${ }^{16}$ porém este é o primeiro relato de isolamento dessa substância na própolis da Bahia.

O espectro de $\mathrm{RMN}{ }^{1} \mathrm{H}$ do composto 7 exibiu sinais que indicam a presença de uma unidade isoprênica devido a presença de um multipleto em $\delta 5,26(1 \mathrm{H})$, característico de hidrogênio vinílico; um dubleto em $\delta 3,38(2 \mathrm{H}, J=6,9)$ atribuído aos hidrogênios alílicos e dois singletos em $\delta 1,87(3 \mathrm{H})$ e $\delta 1,70(3 \mathrm{H})$ compatíveis com as metilas vinílicas. Os sinais referentes a três hidrogênios adjacentes em $\delta 7,80$, $\delta 7,32$ e $\delta 7,26$ corroboram com a presença de um anel aromático $1,2,3$ trisubstituído. Os sinais em $\delta 6,82(1 \mathrm{H}, J=10 \mathrm{~Hz})$ e $5,66(1 \mathrm{H}$, $J=10 \mathrm{~Hz}$ ) são característicos de hidrogênios olefínicos e singleto em $\delta 13,20$ indica a presença de um grupo hidroxila quelado com um grupo carbonila são conclusivos da estrutura da xantona. $\mathrm{O}$ espectro de RMN de ${ }^{13} \mathrm{C}$ coincide com o padrão de sinais do esqueleto básico das xantonas. Através comparação direta dos dados de ${ }^{13} \mathrm{C}$ com os presentes na literatura ${ }^{17}$ foi possível identificar a xantona em questão como sendo a ananixantona (Figura 2), que tem seu primeiro relato de isolamento na própolis. A ananixantona foi anteriormente isolada de Symphonia globulifera uma espécie típica do norte do Brasil que é utilizada como laxante para mulheres grávidas. ${ }^{17}$

\section{Ensaio de sequestro de radicais livres DPPH}

Os antioxidantes são capazes de estabilizar ou desativar os radicais livres antes que ataquem os alvos biológicos nas células. De forma geral, denominam-se antioxidantes as substâncias que presentes em baixas concentrações, comparadas ao substrato oxidável, retardam significativamente ou inibem a oxidação do substrato. ${ }^{18} \mathrm{O}$ ensaio utilizando radical estável DPPH mede a capacidade das substãncias testadas em doar hidrogênio radicalar a este radical. Desta forma, quanto o maior número de hidroxilas presentes na estrutura do composto maior será sua atividade antioxidante.

Os extratos hexânicos e metanólicos não apresentaram atividade oxidante superior a $20 \%$. O extrato hexânico apresentou $\mathrm{IC}_{50}=$ $1,192 \times 10^{-3} \mu \mathrm{g} \mathrm{mL}^{-1}$ enquanto que o extrato metanólico apresentou $\mathrm{IC}_{50}=5,26 \times 10^{-2} \mu \mathrm{g} \mathrm{mL}^{-1}$. O IC $\mathrm{I0}_{50}$ expressa a quantidade de antioxidante necessária para reduzir em 50\% a concentração inicial de DPPH. Este resultado pode ser atribuído da ausência de compostos fenólicos na própolis, visto que o fracionamento inicial dos extratos não levou a identificação ou isolamento de compostos de natureza fenólica e sim de compostos com natureza mais apolar. 


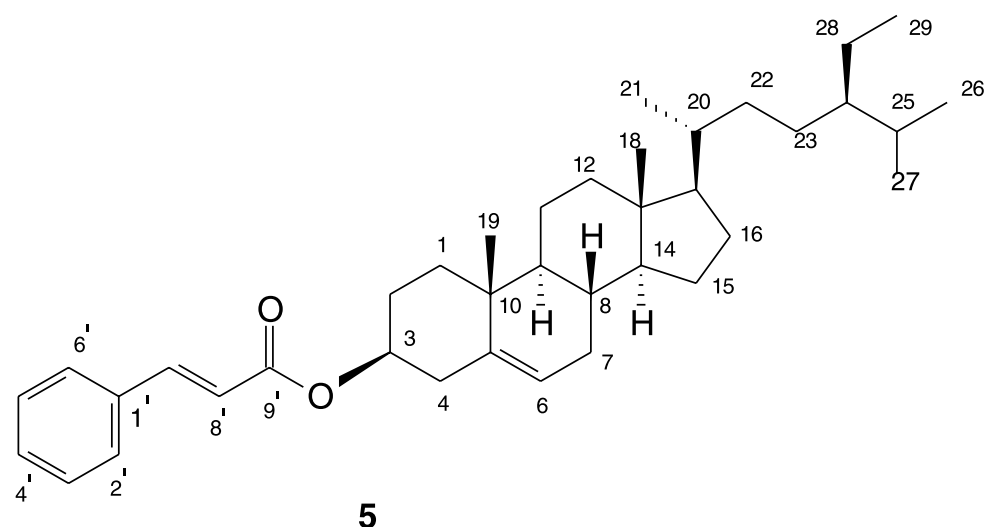<smiles>COC(=O)/C=C/c1ccccc1</smiles><smiles>CC(C)=CCc1c2c(c3oc4c(O)cccc4c(=O)c3c1O)C=CC(C)(C)O2</smiles>

Figura 2. Trans-cinamato de sitosterila (5), cinamato de metila (6) e ananixantona (7) isoladas da própolis da Bahia

\section{Bioensaio de letalidade dos extratos sobre Artemia salina}

O ensaio sobre Artemia salina Leach é um método simples, barato e eficiente para determinação de toxicidade aguda de extratos e substâncias. A Tabela 1 apresenta os resultados de toxicidade para os extratos hexânico (PHC), metanólico (PMC) e para o clorofórmico (PCC). A análise dos resultados obtidos indica que o extrato hexânico apresentou uma baixa toxicidade uma vez que apresentou $\mathrm{DL}_{50}>10^{-3} \mu \mathrm{g} \mathrm{mL} \mathrm{m}^{-1}$. Todavia, os extratos clorofórmico e metanólico apresentaram uma alta toxicidade no teste. O teste de letalidade de $A$. salina apresenta uma correlação com atividade antitumoral e atividade inseticida, podendo ser utilizado como triagem para busca de novos compostos bioativos. ${ }^{12}$ Como a composição química desses extratos ativos ainda não foi estudada, torna-se pertinente uma investigação na composição química buscando isolamento das substâncias responsáveis por essa atividade.

Tabela 1. Resultado dos testes de toxicidade sobre Artemia salina, relativo aos extratos da própolis marrom clara e escura da Bahia

\begin{tabular}{cc}
\hline Extratos & $\mathrm{DL}_{50} \mathrm{mg} / \mathrm{mL}$ \\
\hline PHC & $>1000$ \\
PCC & 68,99 \\
PMC & 118,1 \\
\hline
\end{tabular}

\section{CONCLUSÃO}

O estudo químico do extrato hexânico levou a identificação de quatro hidrocarbonetos o pentadecano, hexadecano, heptadecano e tricosano identificados por CG-EM, o fracionamento cromatográfico do extrato hexânico levou o isolamento dos compostos cinamato de sitosterila e trans cinamato de metila. Até o presente momento do extrato clorofórmico foi isolada a xantona denominada ananixantona.
Estas substâncias ainda não haviam sido isoladas deste tipo de própolis. Os extratos testados não apresentaram uma atividade antioxidante considerável, quando comparado com ácido gálico e/ou quercetina, o que pode ser explicado pela a ausência de compostos fenólicos na própolis do tipo 6. Entretanto, no teste de letalidade de A. salina, os extratos metanólico e clorofórmico apresentaram uma excelente atividade citotóxica que tem boa correlação com atividade tumoral e insesticida.

\section{MATERIAL SUPLEMENTAR}

Algumas imagens dos sistemas e espectros registrados, utilizados neste trabalho estão disponíveis em http://quimicanova.sbq.org.br, na forma de arquivo PDF, com acesso livre.

\section{AGRADECIMENTOS}

A CAPES, CNPq, FABESP/PRONEM e PRONEX/NQAA/ FAPESB pelas bolsas e auxílio financeiro.

\section{REFERÊNCIAS}

1. Ghisalberti, E. L.; Bee Word 1979, 60, 59.

2. Marccuci, M. C.; Quim. Nova 1996, 19, 529.

3. Oldoni, T. L. C.; Dissertação de Mestrado, Universidade do Estado de São Paulo, Brasil, 2007.

4. Pereira, A. S.; Seixas, F. R. M. S.; Neto, F. R. A.; Quim. Nova 2002, 25, 321.

5. Sousa, J. P. B.; Furtado, N. A. J. C.; Jorge, R.; Soares, A. E. E.; Bastos, J. K.; Braz. J. Pharm. 2007, 17, 85.

6. Bankova, V. S.; Castro, S. L.; Marcucci, M. C.; Apidologie 2000, 31, 3.

7. Silici, S.; Kutluca, S.; Ilici, S.; Kutluca, S.; J. Ethnopharmacol. 2005, 99, 69.

8. Kumazawa, S.; Hamazaka, T.; Nakayama, T.; Food Chem. 2004, 84, 329. 
9. Capasso, F.; Castaldo, S.; Fitoterapia. 2002, 73, 1.

10. Lustosa, S. R.; Galindo, B. A.; Nunes, C. C. L.; Randau, P. K; Rolim-Neto, P. J.; Rev. Bras. Farmacogn. 2008, 18, 447.

11. Brand-Williams, W.; Cuvelier, M. E.; Berset, C.; Lebensm. Wiss. Technol. 1995. 22, 25; Alves, C. Q.; David, J. M; David, J. P.; Bahia, M. V.; Aguiar, R. M.; Quim. Nova 2010, 33, 2202.

12. Meyer, B. N.; Ferrigni, N. R.; Jacobsen, L. B.; Nichols, D. E.; McLaughlin, J. L.; Planta Med. 1982, 45, 31; David, J. P.; daSilva, E. F.; deMoura, D. L.; Guedes, M. L. da S., Assunção, R. J.; David, J. M.; Quim. Nova 2001, 24, 730 .
13. Goulart, M. O. F.; Sant'ana, A. E. G.; Lima, R. A.; Cavalcante, S. H.; Carvalho, M. G.; Braz-Filho, R.; Quim. Nova, 1993, 16, 95.

14. Espindola, J. A. A.; Carvalho, G. J. A.; Rodrigues, E. P.; Carvalho, M. G.; Lima, M. E. F.; Souza, S. R.; Floresta Ambient. 2006, 13, 53.

15. Chaloner, A. P.; J. Chem. Soc. 1979, 9, 1028.

16. Ark, Y. K.; Ikegaki, M.; Alencar, S. M.; Honeybee Sci. 2000, 21, 85.

17. Bayma, J. C.; Arruda, M. S. P.; Neto, M. S.; Phytochemistry 1998, 49, 1159.

18. Barreiros, A. L. B. S.; David, J. M.; David, J. P.; Quim. Nova 2006, 29, 113. 